\title{
CHARACTERIZATION AND ANALYSIS OF DOUBLY DISPERSIVE MIMO CHANNELS
}

\author{
Gerald Matz \\ Institute of Communications and Radio-Frequency Engineering, Vienna University of Technology \\ Gusshausstrasse 25/389, A-1040 Vienna, Austria \\ Tel.: +43 1 58801 38916; Fax: +43 158801 38999; Email: gmatz@nt.tuwien.ac.at
}

\begin{abstract}
The characterization, modeling, and simulation of MIMO radio channels is fundamental for the design and evaluation of wireless MIMO systems. While flat-fading (narrowband) MIMO channels are quite well understood, the spatio-temporal-spectral structure of doubly dispersive MIMO channels still poses many problems. In this paper, we view these channels as multivariate WSSUS channels and use tools from (multivariate) spectrum analysis to assess their correlation in space/time/frequency. Our theoretical findings are supported by numerical results involving indoor MIMO measurements.
\end{abstract}

\section{INTRODUCTION}

Multiple-input multiple-output (MIMO) techniques are nowadays considered to be the essential ingredient for high-speed wireless systems [1]. Accurate models for MIMO wireless channels are of central importance for the design and evaluation of such systems. MIMO channel modeling has mostly been restricted to the flat-fading case were only the spatial channel characteristics need to be reproduced (e.g., [2-4]). Much less work deals with frequency-selective [5] or doubly selective MIMO channels [6]. On the other hand, single antenna doubly dispersive channels are by now well understood, the main tools resulting from Bello's wide-sense stationary uncorrelated scattering (WSSUS) theory [7].

In this paper, we attempt to provide a complete statistical characterization of MIMO-WSSUS channels. Particularly, we will argue that two-dimensional (2-D) multivariate spectrum analysis provides the suitable tools to this end. Our approach is useful for assessing the spatio-temporal-spectral correlations present in doubly dispersive MIMO channels. When applied to measurement data, it allows for a variety of conclusions regarding the independence and identicalness of the individual links' statistics and the spatial rank of individual multipath components.

\section{DOUBLY DISPERSIVE MIMO CHANNELS}

The object of our study are doubly dispersive MIMO channels, i.e., channels that feature delays (time dispersion with

This work was supported by the STREP project MASCOT (IST-026905) within the Sixth Framework Programme of the European Commission and by the WWTF project MOHAWI (MA 44). echoes resulting from multipath propagation) and Doppler effects (frequency dispersion resulting from movements of the transmitter/receiver/scatterers). ${ }^{1}$ Throughout the paper, we consider the Rayleigh fading case, i.e., all channel representations are zero-mean and Gaussian.

In a MIMO system with $M$ transmit (TX) antennas and $N$ receive $(\mathrm{RX})$ antennas, the channel $\mathbb{H}$ maps the $M \times 1$ transmit signal $\mathbf{x}(t)$ to the $N \times 1$ receive signal $\mathbf{y}(t)$ as $^{2}$

$$
\mathbf{y}(t)=(\mathbb{H} \mathbf{x})(t)=\int_{\tau} \mathbf{H}(t, \tau) \mathbf{x}(t-\tau) d \tau,
$$

where $\mathbf{H}(t, \tau)$ is the $N \times M$ impulse response matrix ( $\tau$ denotes delay). It is seen from (1) that the channel $\mathbb{H}$ effects time dispersion ( $\tau$-dependence), time selectivity ( $t$-dependence), and spatial dispersion/selectivity (matrix structure). Throughout the paper, the elements of $\mathbf{H}(t, \tau)$ will be referred to as individual links.

The frequency (Doppler) dispersion caused by the channel is better visible in the (matrix-valued) delay-Doppler spreading function (cf. [7] for the single antenna case),

$$
\mathbf{y}(t)=\int_{\tau} \int_{\nu} \mathbf{S}(\tau, \nu)\left(\mathbb{S}_{\tau, \nu} \mathbf{x}\right)(t) d \tau d \nu
$$

Here, $\nu$ denotes Doppler frequency and the action of the timefrequency (delay-Doppler) shift operator $\mathbb{S}_{\tau, \nu}$ is described by $\left(\mathbb{S}_{\tau, \nu} \mathbf{x}\right)(t)=\mathbf{x}(t-\tau) e^{-j 2 \pi \nu t}$. The relation (2) essentially amounts to a representation of the channel as a superposition of weighted time-frequency shifts, the weight being given by $\mathbf{S}(\tau, \nu)$. Hence the spreading function described the timefrequency (doubly) dispersive channel behavior.

In contrast, the time-frequency (doubly) selective fading is more appropriately characterized using the matrix-valued transfer function (again, cf. [7] for the SISO case)

$$
\mathbf{G}(t, f) \triangleq \int_{\tau} \int_{\nu} \mathbf{S}(\tau, \nu) e^{-j 2 \pi(f \tau-t \nu)} d \tau d \nu .
$$

Here, $f$ denotes frequency and $\mathbf{G}(t, f)$ can be interpreted as the MIMO-attenuation of transmit signal components localized about time $t$ and frequency $f$.

\footnotetext{
${ }^{1}$ In this paper, the term "channel" summarizes the effects of the propagation environment, antennas, and RF frontends. All our considerations apply to the equivalent complex baseband.

${ }^{2}$ Integrals are from $-\infty$ to $\infty$ unless stated otherwise.
} 


\section{MIMO-WSSUS CHANNELS}

\subsection{Basic Characterization}

Extending [7], we call a MIMO channel WSSUS if all elements of $\mathbf{G}(t, f)$ are jointly (wide-sense) stationary. Defining $\mathbf{g}(t, f)=\operatorname{vec}\{\mathbf{G}(t, f)\}$, this condition can be written as

$$
\mathcal{E}\left\{\mathbf{g}(t, f) \mathbf{g}^{H}(t+\Delta t, f+\Delta f)\right\}=\mathbf{R}(\Delta t, \Delta f) .
$$

Here, $\mathcal{E}\{\cdot\}$ denotes expectation (ensemble average) and $\mathbf{R}(\Delta t, \Delta f)$ is is referred to as the spatio-temporal-spectral matrix correlation function (dimension $N M \times N M$ ) of the the channel. It describes the correlations of any two component transfer functions $G_{n, m}(t, f)$ and $G_{n^{\prime}, m^{\prime}}\left(t^{\prime}, f^{\prime}\right)$ separated by $t^{\prime}-t=\Delta t$ in time and by $f^{\prime}-f=\Delta f$ in frequency.

Equivalently, the MIMO-WSSUS property requires that the elements of $\mathbf{S}(\tau, \nu)$ are jointly (wide-sense) white, i.e.,

$$
\mathcal{E}\left\{\mathbf{s}(\tau, \nu) \mathbf{s}^{H}\left(\tau^{\prime}, \nu^{\prime}\right)\right\}=\mathbf{C}(\tau, \nu) \delta\left(\tau-\tau^{\prime}\right) \delta\left(\nu-\nu^{\prime}\right)
$$

with $\mathbf{s}(\tau, \nu)=\operatorname{vec}\{\mathbf{S}(\tau, \nu)\}$. The $N \times M$ matrix $\mathbf{C}(\tau, \nu)$ will be referred to as scattering function matrix (SFM). The SFM is positive semi-definite for all $(\tau, \nu)$. It summarizes the spatial characteristics and strength of all scatterers (more precisely, multipath components) with delay $\tau$ and Doppler $\nu$. The SFM can be shown to be the 2-D Fourier transform of the spatio-temporal-spectral correlation,

$\mathbf{C}(\tau, \nu)=\int_{\Delta t} \int_{\Delta f} \mathbf{R}(\Delta t, \Delta f) e^{-j 2 \pi(\nu \Delta t-\tau \Delta f)} d \Delta t d \Delta f$.

Thus, the SFM can be interpreted as the 2-D power spectral matrix of the 2-D stationary multivariate random process $\mathrm{g}(t, f)$, consisting of auto-scattering functions $C_{k, k}(\tau, \nu)$, $k=1, \ldots, N M$, for the individual links and cross-scattering functions $C_{k, l}(\tau, \nu)=C_{l, k}^{*}(\tau, \nu), k \neq l$ (here, $C_{k, l}(\tau, \nu)$ denotes the SFM element in the $k$ th row and lth column). This interpretation suggests that the statistical characterization of MIMO-WSSUS channels is in fact a spectrum analysis problem. Much of the subsequent discussion will be based on this observation.

\subsection{Canonical Channel Decomposition}

Consider the eigendecomposition of the SFM,

$$
\mathbf{C}(\tau, \nu)=\sum_{n=1}^{N} \sum_{m=1}^{M} \lambda_{n, m}(\tau, \nu) \mathbf{u}_{n, m}(\tau, \nu) \mathbf{u}_{n, m}^{H}(\tau, \nu),
$$

where $\lambda_{n, m}(\tau, \nu) \geq 0$ and $\mathbf{u}_{n, m}(\tau, \nu)$ denote the eigenvalues and eigenvectors of $\mathbf{C}(\tau, \nu)$, respectively (we use double indices for later convenience). For each $(\tau, \nu)$, the $N M$ vectors $\mathbf{u}_{n, m}(\tau, \nu)$ form an orthonormal basis of $\mathbb{C}^{N M}$. These bases allow to expand the channel's spreading function as

$$
\mathbf{S}(\tau, \nu)=\sum_{n=1}^{N} \sum_{m=1}^{M} \alpha_{n, m}(\tau, \nu) \mathbf{U}_{n, m}(\tau, \nu)
$$

where $\mathbf{U}_{n, m}(\tau, \nu)=\operatorname{unvec}\left\{\mathbf{u}_{n, m}(\tau, \nu)\right\}$ and

$$
\alpha_{n, m}(\tau, \nu)=\mathbf{u}_{n, m}^{H}(\tau, \nu) \mathbf{s}(\tau, \nu) .
$$

Their correlation of these coefficients equals

$$
\begin{aligned}
\mathcal{E}\{ & \left.\alpha_{n, m}(\tau, \nu) \alpha_{n^{\prime}, m^{\prime}}^{*}\left(\tau^{\prime}, \nu^{\prime}\right)\right\} \\
& =\mathbf{u}_{n, m}^{H}(\tau, \nu) \mathcal{E}\left\{\mathbf{s}(\tau, \nu) \mathbf{s}^{H}\left(\tau^{\prime}, \nu^{\prime}\right)\right\} \mathbf{u}_{n^{\prime}, m^{\prime}}\left(\tau^{\prime}, \nu^{\prime}\right) \\
& =\mathbf{u}_{n, m}^{H}(\tau, \nu) \mathbf{C}(\tau, \nu) \delta\left(\tau-\tau^{\prime}\right) \delta\left(\nu-\nu^{\prime}\right) \mathbf{u}_{n^{\prime}, m^{\prime}}\left(\tau^{\prime}, \nu^{\prime}\right) \\
& =\mathbf{u}_{n, m}^{H}(\tau, \nu) \mathbf{C}(\tau, \nu) \mathbf{u}_{n^{\prime}, m^{\prime}}\left(\tau^{\prime}, \nu^{\prime}\right) \delta\left(\tau-\tau^{\prime}\right) \delta\left(\nu-\nu^{\prime}\right) \\
& =\lambda_{n, m}(\tau, \nu) \delta_{n, n^{\prime}} \delta_{m, m^{\prime}} \delta\left(\tau-\tau^{\prime}\right) \delta\left(\nu-\nu^{\prime}\right),
\end{aligned}
$$

where we used the WSSUS property and the fact that the eigenvectors diagonalize the SFM. It is seen, that the coefficients $\alpha_{n, m}(\tau, \nu)$ are uncorrelated with respect to delay, Doppler, and space. The expansion (4) entails the following channel representation:

$$
\mathbb{H}=\int_{\tau} \int_{\nu} \sum_{n=1}^{N} \sum_{m=1}^{M} \alpha_{n, m}(\tau, \nu) \mathbf{U}_{n, m}(\tau, \nu) \mathbb{S}_{\tau, \nu}
$$

Here, $\mathbb{U}_{n, m}(\tau, \nu) \triangleq \mathbf{U}_{n, m}(\tau, \nu) \mathbb{S}_{\tau, \nu}$ denotes deterministic atomic MIMO channels that effect a delay by $\tau$, a Doppler shift $\nu$, and a spatial TX/RX weighting $\mathbf{U}_{n, m}(\tau, \nu)$. A closer look reveals that these atomic channels themselves are spacetime-frequency orthogonal with respect to the matrix-operator inner product, i.e.,

$$
\begin{aligned}
\left\langle\left\langle\mathbb{U}_{n, m}\right.\right. & \left.\left.(\tau, \nu), \mathbb{U}_{n^{\prime}, m^{\prime}}\left(\tau^{\prime}, \nu^{\prime}\right)\right\rangle\right\rangle \\
& \triangleq \operatorname{tr}\left\{\mathbf{U}_{n, m}(\tau, \nu)\left\langle\mathbb{S}_{\tau, \nu}, \mathbb{S}_{\tau^{\prime}, \nu^{\prime}}\right\rangle \mathbf{U}_{n^{\prime}, m^{\prime}}^{H}\left(\tau^{\prime}, \nu^{\prime}\right)\right\} \\
& =\delta_{n, n^{\prime}} \delta_{m, m^{\prime}} \delta\left(\tau-\tau^{\prime}\right) \delta\left(\nu-\nu^{\prime}\right) .
\end{aligned}
$$

The expansion (5) is thus doubly orthogonal in the sense that the atomic channels are deterministically orthogonal and the associated coefficients are statistically orthogonal. The significance of (5) comes from the fact that it decomposes any MIMO-WSSUS channel into a superposition of simple deterministic atomic MIMO channels weighted with uncorrelated scalar random coefficients. Hence, the channel transfer effects (delay-Doppler shifts and spatial signatures) are separated from the channel stochastics.

\subsection{Special Cases}

We next discuss special cases of MIMO-WSSUS channels that extend corresponding flat-fading counterparts $[2,3,6]$.

i.i.d. Model. Here, $\mathbf{C}(\tau, \nu)=C(\tau, \nu)$ I, i.e., all elements of $\mathbf{H}(t, \tau)$ are independent WSSUS channels with identical scattering function $C(\tau, \nu)$. In this case, $\lambda_{n, m}(\tau, \nu)=$ $C(\tau, \nu)$ and $\mathbf{U}_{n, m}(\tau, \nu)=\mathbf{e}_{n} \mathbf{e}_{m}^{H}$, with $\mathbf{e}_{n}$ denoting the $n$th unit vector of appropriate dimension. Here, the action of the atomic channels is $\left(\mathbb{U}_{n, m}(\tau, \nu) \mathbf{x}\right)(t)=x_{m}(t-\tau) e^{j 2 \pi \nu t} \mathbf{e}_{n}$.

Kronecker Model. Here, the SFM eigenmatrices are rank-one, $\mathbf{U}_{n, m}(\tau, \nu)=\mathbf{v}_{n}(\tau, \nu) \mathbf{w}_{m}^{H}(\tau, \nu)$, and the SFM eigenvalues are separable w.r.t. the TX and RX antenna indices, $\lambda_{n, m}(\tau, \nu)=\kappa_{n}(\tau, \nu) \mu_{m}(\tau, \nu)$. This means that 
the atomic channels are characterized by spatial TX/RX signatures $\mathbf{v}_{n}(\tau, \nu), \mathbf{w}_{m}^{H}(\tau, \nu)$ with respective average powers $\kappa_{n}(\tau, \nu), \mu_{m}(\tau, \nu)$.

Weichselberger Model. This model differs from the Kronecker model in that the powers $\lambda_{n, m}(\tau, \nu)$ of the eigenmodes $\mathbf{U}_{n, m}(\tau, \nu)=\mathbf{v}_{n}(\tau, \nu) \mathbf{w}_{m}^{H}(\tau, \nu)$ are not separable. The resulting simplified version of (4) can be written as

$$
\mathbf{S}(\tau, \nu)=\mathbf{V}(\tau, \nu)[\boldsymbol{\Omega}(\tau, \nu) \odot \boldsymbol{\Gamma}(\tau, \nu)] \mathbf{W}^{H}(\tau, \nu),
$$

with the matrices $\mathbf{V}(\tau, \nu)=\left[\mathbf{v}_{1}(\tau, \nu) \ldots \mathbf{v}_{N}(\tau, \nu)\right]$, $\mathbf{W}(\tau, \nu)=\left[\mathbf{w}_{1}(\tau, \nu) \ldots \mathbf{w}_{m}(\tau, \nu)\right]$, and $[\boldsymbol{\Omega}(\tau, \nu)]_{n, m}=$ $\sqrt{\lambda_{n, m}(\tau, \nu)}$; furthermore, $\boldsymbol{\Gamma}(\tau, \nu)$ is an i.i.d. Gaussian random matrix and $\odot$ denotes the Hadamard product.

Virtual MIMO Model. The virtual MIMO model is a special case of the Weichselberger model for uniform linear array (ULAs); it assumes that the spatial signatures equal the array steering vectors, i.e., $\mathbf{V}(\tau, \nu)=\mathbf{F}_{N}$ and $\mathbf{W}(\tau, \nu)=$ $\mathbf{F}_{M}$, where $\mathbf{F}_{N}$ denotes the $N \times N$ DFT matrix.

\subsection{Numerical Results}

To illustrate the usefulness of the SFM and the canonical channel decomposition, we use indoor measurement data obtained in the course of a channel sounding campaign at Vienna Airport (cf. [8]). The equipment consisted of an Elektrobit PropSound channel sounder with $2.45 \mathrm{GHz}$ carrier frequency and $200 \mathrm{MHz}$ bandwidth. At the TX side, a horizontally omni-directional 7+1 circular monopole array with vertical polarization was used whereas at the RX side a dualpolarized $4 \times 4$ ULA of patch antennas was employed.

We picked a measurement in the check-in hall and selected a $4 \times 1$ SIMO subsystem consisting of the central TX antenna and the first row of the RX array. Using a single channel realization consisting of 128 channel impulse response snapshots, we estimated the SFM using 2-D multi-window spectrum estimators $[9,10]$. The resulting SFM estimate (in $\mathrm{dB}$ ) is shown in Fig. 1, with darker shading corresponding to larger values. The $n$th row and $n^{\prime}$ th column shows the (cross)scattering function of $[\mathbf{H}(t, \tau)]_{n, 1}$ and $[\mathbf{H}(t, \tau)]_{n^{\prime}, 1}$.

It is seen that for this measurement, the SFM features a small number of well-localized delay-Doppler components. Furthermore, the diagonal SFM elements are very similar, indicating that all $[\mathbf{H}(t, \tau)]_{n, 1}, n=1 \ldots, 4$, are identically distributed. Finally, the large off-diagonal SFM elements suggest strong spatial correlations of the individual delayDoppler components.

To analyze the spatial structure of the SFM in more detail, we calculated the eigenvalues and eigenvectors of $\mathbf{C}(\tau, \nu)$ for the delay-Doppler coordinates $\left(\tau_{i}, \nu_{i}\right), i=1, \ldots, 4$, corresponding to the four strongest components. The corresponding eigenvalue spreads $\frac{\left[\sum_{n, m} \lambda_{n, m}\left(\tau_{i}, \nu_{i}\right)\right]^{2}}{\sum_{n, m} \lambda_{n, m}^{2}\left(\tau_{i}, \nu_{i}\right)}$ were 1.05 , $1.2,1.22$, and 1.45 , i.e., the SFM had essentially rank one for all four components. Repeating this experiment with various other measurement data lead to the conclusion that for strong,

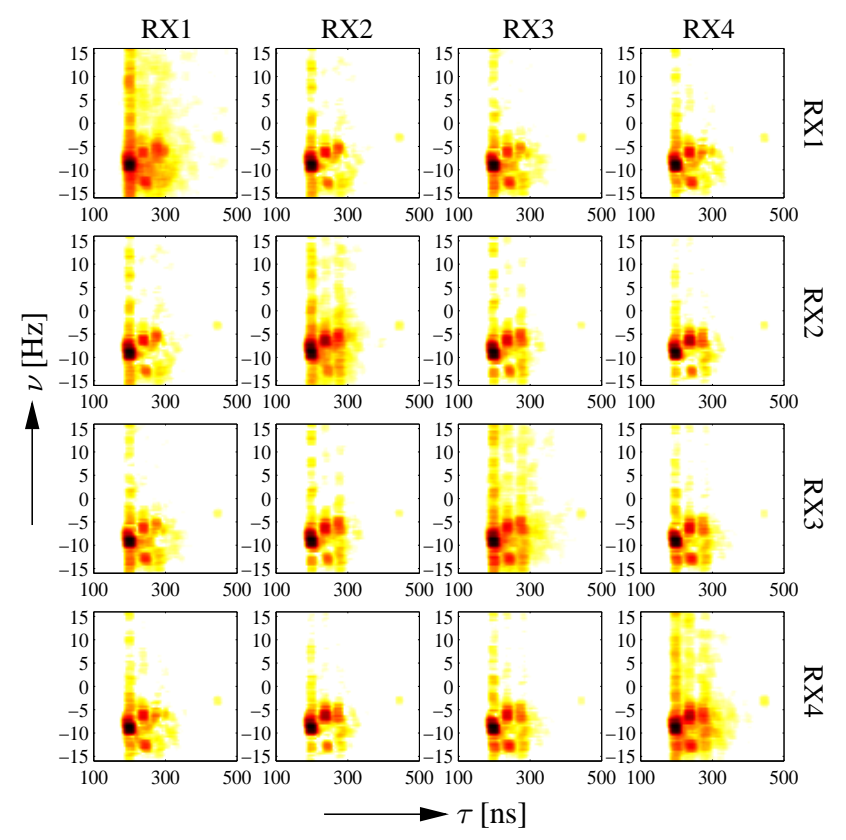

Fig. 1. Estimated SFM for a $4 \times 1$ SIMO system.

isolated (i.e., specular) delay-Doppler components the SFM is indeed approximately rank-one. Furthermore, in most of the cases the associated eigenmatrix had approximately rank one as well, i.e., the channel is essentially rank-one Kronecker. This is intuitive since specular components usually have welldefined angles of departure and arrival. Assuming $P$ purely specular components, the observed rank-one Kronecker property simplifies (4) to

$$
\mathbf{S}(\tau, \nu)=\sum_{i} \alpha_{i} \mathbf{v}_{i} \mathbf{w}_{i}^{H} \delta\left(\tau-\tau_{i}\right) \delta\left(\nu-\nu_{i}\right) .
$$

We emphasize that the above considerations do not apply to diffuse scattering components.

Our observations above seemingly contradict earlier work [11] that proved the Kronecker model wrong. However, there the spatial correlations in the TF domain have been considered, which equal (cf. (3))

$$
\mathbf{R}(0,0)=\mathcal{E}\left\{\mathbf{g}(t, f) \mathbf{g}^{H}(t, f)\right\}=\int_{\tau} \int_{\nu} \mathbf{C}(\tau, \nu) d \tau d \nu .
$$

Hence, even if $\mathbf{C}(\tau, \nu)$ is rank-one Kronecker, the delayDoppler averaging typically builds up full rank for $\mathbf{R}(0,0)$.

\section{SF SIMILARITY}

\subsection{Distance Measures}

In the i.i.d. model, all $[\mathbf{H}(t, \tau)]_{n, m}$ have the same scattering function (SF). In Subsection 3.4, we saw measurement data for which this property appeared to be true. For a systematic empirical assessment of this property, we need appropriate (dis)similarity measures for two SFs $C_{1}(\tau, \nu)$ and $C_{2}(\tau, \nu)$. 


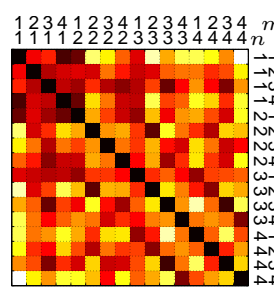

(a)

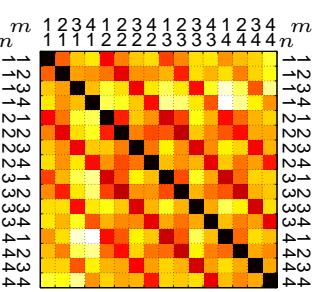

(c)
Fig. 2. SF similarities of all 256 link pairs in a $4 \times 4$ MIMO system: (a) $\gamma_{1}$, (b) $\gamma_{2}^{\prime}$, and (c) $\gamma_{3}^{\prime}$, with white corresponding to minimum similarity of 0.78 and black corresponding to 1 .

One approach is to view SFs as elements of the Hilbert space $L^{2}\left(\mathbb{R}^{2}\right)$ and measure similarity in terms of collinearity via the normalized inner product

$$
\gamma_{1} \triangleq \frac{\left\langle C_{1}, C_{2}\right\rangle}{\left\|C_{1}\right\|\left\|C_{2}\right\|}
$$

We have $\gamma_{1} \leq 1$, with equality iff $C_{1}(\tau, \nu)=c C_{2}(\tau, \nu)$ for some constant $c$. Furthermore, $\gamma_{1} \geq 0$, with equality iff the two SFs are orthogonal. Since SFs are nonnegative, the latter can occur only if $C_{1}(\tau, \nu)$ and $C_{2}(\tau, \nu)$ have disjoint support, a restriction that appears somewhat undesirable.

Hence, we take the non-negativity of the SFs into account and view them as elements of an appropriate Riemannian manifold. "Distances" on such manifolds are usually defined in terms of divergences. A suitable divergence that is wellknown from statistics and information theory is given by the Kullback-Leibler divergence (KLD) [12],

$$
\gamma_{2} \triangleq \int_{\tau} \int_{\nu} \tilde{C}_{1}(\tau, \nu) \log \frac{\tilde{C}_{1}(\tau, \nu)}{\tilde{C}_{2}(\tau, \nu)} d \tau d \nu,
$$

where $\tilde{C}_{i}(\tau, \nu)=C_{i}(\tau, \nu) / \int_{\tau} \int_{\nu} C_{i}(\tau, \nu) d \tau d \nu$. However, the KLD might still not be perfectly suited since it was originally defined for probability density functions, which necessitates the additional normalization in (6). An alternative divergence measure for positive, non-normalized spectral densities has been recently proposed in [13] and reads

$$
\gamma_{3} \triangleq \log \left(\frac{1}{|\mathcal{S}|} \iint_{\mathcal{S}} \frac{C_{1}(\tau, \nu)}{C_{2}(\tau, \nu)} d \tau d \nu \frac{1}{|\mathcal{S}|} \iint_{\mathcal{S}} \frac{C_{2}(\tau, \nu)}{C_{1}(\tau, \nu)} d \tau d \nu\right),
$$

where $\mathcal{S}$ denotes the SF support region and $|\mathcal{S}|$ is its size. We have $\gamma_{2} \geq 0$ and $\gamma_{3} \geq 0$ with equality in both cases iff $C_{1}(\tau, \nu)=c C_{2}(\tau, \nu)$.

\subsection{Numerical Example}

We applied our (dis)similarity measures to a different set of channel measurements from Vienna Airport. This time, we consider a MIMO subsystem with $4 \mathrm{TX}$ and $4 \mathrm{RX}$ antennas. We estimated the SFs of the corresponding 16 individual links (i.e., the 16 diagonal elements of the SFM), and calculated $\gamma_{1}$, $\gamma_{2}$, and $\gamma_{3}$ for all possible SF pairs. The results are shown in Fig. $2\left(\gamma_{2}\right.$ and $\gamma_{3}$ were compressed to the interval $[0,1]$ via the transformation $\gamma_{i}^{\prime} \rightarrow \frac{2}{2+\gamma_{i}}, i=1,2$, to make them comparable with $\gamma_{1}$ ). All results confirm that the 16 links have strongly similar SFs (the average similarities were $\bar{\gamma}_{1}=0.89$, $\bar{\gamma}_{2}^{\prime}=0.90$, and $\bar{\gamma}_{3}^{\prime}=0.86$ ). This similarity is slightly more pronounced on the RX side as indicated by the the slightly larger results on every fourth diagonal in Fig. 2 (these correspond to identical TX antenna indices). This phenomenon can be explained by the fact that the TX antenna spacing was larger than the RX antenna spacing. Comparing the three similarity measures, $\gamma_{2}^{\prime}$ and $\gamma_{3}^{\prime}$ appear to be more suitable than $\gamma_{1}$. In particular, $\gamma_{3}^{\prime}$ yields more homogeneous results and reflects the increased RX similarity more consistently.

\section{COHERENCE ANALYSIS}

\subsection{Delay-Doppler Coherence}

The second assumption in the i.i.d. model pertains to the independence of the elements of the MIMO matrix. In Subsection 3.4 , we have already seen an example where this assumption is violated. However, the cross-SF of two links per se does not allow to judge the amount of correlation.

Again, we will borrow tools from (cross-)spectral analysis to resolve this issue. In particular, the correlation of two stationary processes is most clearly studied in terms of their (spectral) coherence function [14]. We thus defined the delayDoppler coherence (DDC) of the $k$ th and $l$ th link as ${ }^{3}$

$$
\Gamma_{k, l}(\tau, \nu) \triangleq \frac{C_{k, l}(\tau, \nu)}{\sqrt{C_{k, k}(\tau, \nu) C_{l, l}(\tau, \nu)}}, \quad k \neq l .
$$

The DDC matrix $\boldsymbol{\Gamma}(\tau, \nu)$ of a MIMO-WSSUS channel is defined in terms of its elements as

$$
[\boldsymbol{\Gamma}(\tau, \nu)]_{k, l} \triangleq \begin{cases}\Gamma_{k, l}(\tau, \nu), & k \neq l \\ C_{k, k}(\tau, \nu), & k=l\end{cases}
$$

It can easily be seen that this definition ensures that the SFM $\mathbf{C}(\tau, \nu)$ and the DDC matrix $\boldsymbol{\Gamma}(\tau, \nu)$ are in one-to-one correspondence. The DDC satisfies

$$
0 \leq\left|\Gamma_{k, l}(\tau, \nu)\right| \leq 1
$$

If $\Gamma_{k, l}(\tau, \nu)=0$ for a certain $(\tau, \nu)$, then the corresponding delay-Doppler components of the $k$ and $l$ th link, $[\mathbf{s}(\tau, \nu)]_{k}$ and $[\mathbf{s}(\tau, \nu)]_{l}$, are totally uncorrelated; if $\left|\Gamma_{k, l}(\tau, \nu)\right|=1$, then the delay-Doppler components feature maximum correlation in the sense that they are linearly dependent, i.e., for that $(\tau, \nu),[\mathbf{s}(\tau, \nu)]_{k}=c[\mathbf{s}(\tau, \nu)]_{l}$ with a fixed constant $c$.

\subsection{Numerical Results}

We next provide an example for the DDC. by considering a $2 \times 1$ SIMO subsystem of the airport data. We estimated the SFM using multi-window spectrum estimation techniques and calculated the DDC according to (7). The result is shown

\footnotetext{
${ }^{3}$ Sometimes, the term "coherence" is used for the magnitude or magnitude squared of our definition.
} 

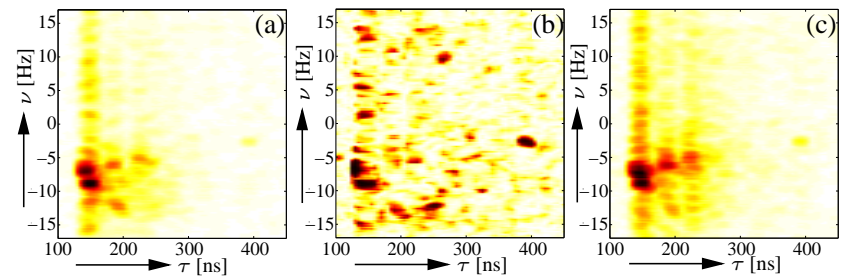

Fig. 3. Coherence analysis of a measured channel: $C_{11}(\tau, \nu)$ in $d B,(b)\left|\Gamma_{1,2}(\tau, \nu)\right|,(b) C_{22}(\tau, \nu)$ in $d B$.

in Fig. 3. It can be seen that most of the dominant components are strongly coherent. The DDC even reveals a weak, but strongly coherent component at $(390 \mathrm{~ns},-3 \mathrm{~Hz})$. For some components, however, $\left|\Gamma_{1,2}(\tau, \nu)\right| \ll 1$; this either means that the respective delay-Doppler components are indeed incoherent, or indicates that our assumptions regarding the joint WSSUS property of the two links are violated. To resolve this question requires tools for non-WSSUS MIMO channels that are beyond the scope of this paper.

An estimated DDC matrix (obtained in a similar way as before) for a $2 \times 2$ MIMO system measured in the shopping arcade of Vienna Airport is shown in Fig. 4. Inspecting the diagonal reveals that again all individual SFs are highly similar. Furthermore, these SFs were found to be approximately separable with a Gaussian-mixture-type Doppler profile and an exponential delay profile. Inspecting the off-diagonals reveals that there is reasonable coherence of the essential delayDoppler components. This coherence is more pronounced for $\Gamma_{1,3}(\tau, \nu)$ and $\Gamma_{2,4}(\tau, \nu)$, which correspond to channel pairs with identical TX antenna but different RX antenna. We conclude that RX coherence is stronger than TX coherence in this example, which can again be attributed to the smaller antenna spacing at the RX side.

\section{CONCLUSION}

In this paper, we considered MIMO-WSSUS channels and particularly advocated the use of two-dimensional spectrum analysis techniques for their characterization and analysis. This lead to some new tools for doubly dispersive MIMO channel characterization: the scattering function matrix, the coherence matrix, and appropriate similarity measures for scattering functions. Numerical examples involving indoor MIMO measurements illustrated our ideas and revealed that isolated delay-Doppler components can typically be well approximated via low-rank spatial models; furthermore, antenna spacing was seen to have a critical impact on the statistical similarity and correlation of the individual MIMO elements.

\section{REFERENCES}

[1] A. Paulraj, R. U. Nabar, and D. Gore, Introduction to SpaceTime Wireless Communications, Cambridge Univ. Press, Cambridge (UK), 2003.

[2] W. Weichselberger, M. Herdin, H. Özcelik, and E. Bonek, “A stochastic MIMO channel model with joint correlation of both

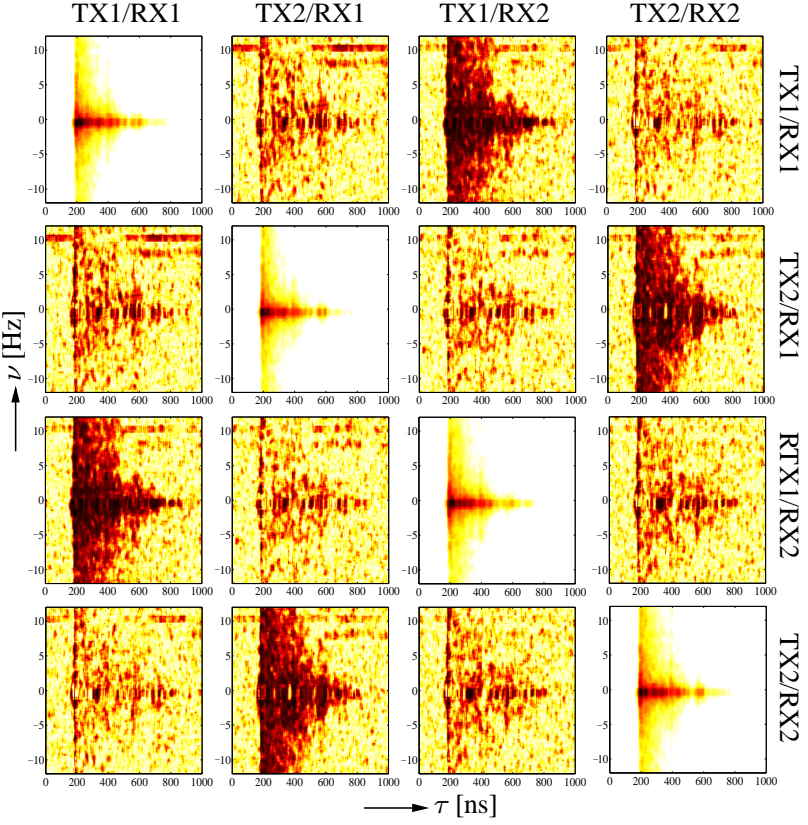

Fig. 4. Estimated DDC matrix for a $2 \times 2$ MIMO system.

link ends," IEEE Trans. Wireless Comm., vol. 5, no. 1, pp. 90-100, Jan. 2006.

[3] J.P. Kermoal, L. Schumacher, K.I. Pedersen, P.E. Mogensen, and F. Frederiksen, "A stochastic MIMO radio channel model with experimental validation," IEEE J. Sel. Ar. Comm., vol. 20, no. 6, pp. 1211-1226, Aug. 2002.

[4] D. Gesbert, H. Bölcskei, D. A. Gore, and A. J. Paulraj, "Outdoor MIMO wireless channels: Models and performance prediction," IEEE Trans. Comm., vol. 50, no. 12, pp. 1926-1934, Dec. 2002, submitted.

[5] H. Bölcskei, D. Gesbert, and A. J. Paulraj, "On the capacity of OFDM-based spatial multiplexing systems," IEEE Trans. Comm., vol. 50, no. 2, pp. 225-234, Feb. 2002.

[6] A. Sayeed, "A virtual representation for time- and frequencyselective correlated MIMO channels," in Proc. IEEE ICASSP03, Hong Kong, April 2003.

[7] P. A. Bello, "Characterization of randomly time-variant linear channels," IEEE Trans. Comm. Syst., vol. 11, pp. 360-393, 1963.

[8] Markus Herdin, Non-Stationary Indoor MIMO Radio Channels, Ph.D. thesis, Vienna University of Technology, 2004.

[9] D. J. Thomson, "Spectrum estimation and harmonic analysis," Proc. IEEE, vol. 70, no. 9, pp. 1055-1096, 1982.

[10] G. Matz, "Doubly underspread non-WSSUS channels: Analysis and estimation of channel statistics," in Proc. IEEE SPAWC-03, Rome, Italy, June 2003, pp. 190-194.

[11] H. Özcelik, M. Herdin, W. Weichselberger, J. Wallace, and E. Bonek, "Deficiencies of Kronecker MIMO radio channel model," Electronics Letters, vol. 39, no. 16, pp. 1209-1210, 2003.

[12] T. M. Cover and J. A. Thomas, Elements of Information Theory, Wiley, New York, 1991.

[13] Tryphon T. Georgiou, "Distances between power spectral densities," arXiv:math.OC/0607026.

[14] J. S. Bendat and A. G. Piersol, Engineering Applications of Correlation and Spectral Analysis, Wiley, New York, 2nd edition, 1993. 